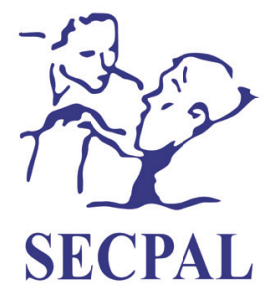

Medicina Paliativa

www.medicinapaliativa.es

ORIGINAL

\title{
Estudio Palidem: cuidados al final de la vida en pacientes con demencia avanzada institucionalizados
}

\author{
Javier Alaba*1,3, Enrique Arriola ${ }^{2,3}$, Iván Antón ${ }^{3}$, Álvaro García-Soler ${ }^{3}$, Cristina Buiza $^{3}$ y \\ Carmen Hernández ${ }^{3}$
}

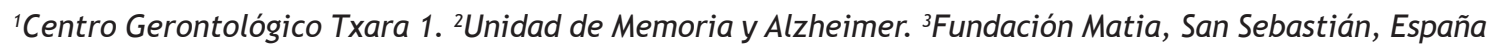

Recibido el 16 de marzo de 2018

Aceptado el 29 de junio de 2018

\section{PALABRAS CLAVE \\ Demencia, cuidados paliativos, centros gerontológicos.}

\begin{abstract}
Resumen
Objetivo: Desarrollar un plan de atención específico en pacientes con demencia avanzada institucionalizados, planificando los cuidados mediante el control sintomático, tomando decisiones con el cuidador principal, realizando un apoyo psicológico y espiritual.

Método: Estudio descriptivo de periodo en el Centro Txara 1, desde el 1 de julio de 2014 hasta el 31 de diciembre de 2016, identificando a aquellos residentes con demencia avanzada y síndrome de declive. Se recoge trimestralmente el grado de control de síntomas (Symptom Management at the End-of-Life in Dementia) (SM-EOLD), utilizando la escala PAINAD para la valoración del dolor; el confort en el momento de la agonía se determina diariamente (The Comfort Assessment in Dying with Dementia) (CAD-EOLD), estableciendo con el cuidador principal la toma de decisiones basadas en los objetivos asistenciales paliativos. Tras el fallecimiento se realiza una encuesta de satisfacción al cuidador (Satisfaction with Care at the End-of-Life in Dementia) (SWC-EOLD) y contacto periódico con la pastoral para el apoyo espiritual.
\end{abstract}

*Autor para correspondencia:

Javier Alaba

Paseo de los Pinos 35, 20018, San Sebastián

Correo electrónico: javier.alaba@matiafundazioa.net

DOI: $10.20986 /$ medpal.2019.1061/2019

1134-248X/ ( 2019 Sociedad Española de Cuidados Paliativos. Publicado por Inspira Network. Todos los derechos reservados. 
Resultados: Se han analizado 74 residentes, con una edad media de 87 años, un $79 \%$ mujeres, con 4,6 medicamentos de media, con enfermedad de Alzheimer como principal causa de demencia. En el control de síntomas en fase estable no se detecta inestabilidad física o conductual relevante, detectándose en un $5 \%$ la presencia de dolor. De 50 residentes fallecidos, el $94 \%$ sucede en el propio centro, en un $67 \%$ se puede registrar el grado de confort destacando la presencia de disnea y disfagia, al ser la infección respiratoria aspirativa la principal causa de fallecimiento. En un $66 \%$ de la población seleccionada se realiza atención espiritual. Un 30 \% de los cuidadores principales responden a la encuesta de satisfacción, con un alto porcentaje de aceptación en el nivel de cuidados.

Conclusiones: La identificación de los pacientes con demencia avanzada en situación de final de vida permite estructurar un plan de atención en el centro gerontológico, conociendo las necesidades sintomáticas, el grado de confort o bienestar y consensuando las decisiones en cuanto a la proporcionalidad de cuidados con la familia. Ante situaciones de descompensación aguda, la coordinación con los recursos sociosanitarios disponibles es fundamental para poder llevar a cabo las decisiones adoptadas del equipo sanitario con el cuidador principal.

\section{KEYWORDS}

Dementia, hospice, palliative care nursing.

\begin{abstract}
Objective: To develop a specific care plan for institutionalized patients with advanced dementia based on symptom control and including joint decision making with the primary caregiver as well as psychological and spiritual support.

Method: A descriptive 1-period study at Centro Txara 1, from July 12014 to December 312016 , which identified residents with advanced dementia and geriatric failure-to-thrive syndrome. Symtom control extent was quarterly assessed using the Symptom Management at the End-ofLife in Dementia (SM-EOLD) scale, and the PAINAD scale for pain; comfort at the end of life was assessed daily with the Comfort Assessment in Dying with Dementia (CAD-EOLD) instrument, and decisions were made jointly with the primary caregiver based on the goals of palliative care. After the death of the patient a satisfaction survey was administered to the caregiver (Satisfaction with Care at the End-of-Life in Dementia, SWC-EOLD), who was also provided with regular contact with the pastoral care team for spiritual support.
\end{abstract}

Results: A total of 74 residents were assessed with a mean age of 87 years; $79 \%$ were females, mean number of drugs was 4.6, and Alzheimer's disease was the primary cause of dementia. Stable-phase symptom control identified no relevant phisical or behavioral instability, but pain was found in $5 \%$ of the sample. Of 50 residents who passed away, $94 \%$ died in the hospice; in $67 \%$ comfort degree could be collected, and the presence of shortness of breath and dysphagia stood out, as aspiration-induced respiratory infection was the primary cause of death. Spiritual care was provided for $66 \%$ of the study population. In all, $30 \%$ of primary caregivers responded to the satisfaction survey showing a high percentage of acceptance of level of care.

Conclusions: Identifying patients with advanced dementia at the end of life allows the development of a hospice care plan considering symptom needs, comfort degree and wellbeing, and involving consensus decisions about care proportionality with the family. Should an acute decompensation occur, coordination with the available social and healthcare resources is key for implementing the decisions agreed between the healthcare team and the primary caregiver.

Alaba J, Arriola E, Antón I, García-Soler A, Buiza C, Hernández C. Estudio Palidem: cuidados al final de la vida en pacientes con demencia avanzada institucionalizados. Med Paliat. 2019;26(2):143-149.

\section{Introducción}

Se estima que en España, en el año 2014, había unos 5390 centros residenciales, que atendían a unas 351.548 personas $^{1}$. La demencia constituye una de las principales causas de institucionalización y de dependencia, ya que aproximadamente un $60 \%$ de la población geriátrica institucionalizada presenta un síndrome demencial, siendo la enfermedad de Alzheimer la más prevalente ${ }^{2}$.
Según datos de residentes atendidos en el año 2016 por la Fundación Matia (FM), que engloba a siete centros gerontológicos, un 70 \% de los residentes fallecieron en los mismos centros. La localización del fallecimiento del paciente aporta poca información sobre los aspectos cualitativos del proceso de final de vida, por lo que es de gran relevancia evaluar las necesidades asistenciales que vayan surgiendo en fase de estabilidad y ante situaciones de crisis. 
Desde hace tiempo se conocen las trayectorias y necesidades de las personas con demencia en situación de final de vida, principalmente en centros gerontológicos o recursos de larga estancia. El grupo de investigación de la Dra. Mitchell y colaboradores en Boston fue pionero en estructurar dicha atención. Así, en el año 2004 se publicó el estudio CASCADE (Choices-opciones, Attitudes-actitudes, Strategiesestrategias, Care-cuidados, Advanced-avanzada, Dementiademencia, End of live-final de la vida), en el que se mide el control de síntomas, el grado de confort en los momentos de la agonía y la evaluación del grado de satisfacción del representante familiar con los cuidados instaurados ${ }^{3-7}$.

En estudios sobre planificación avanzada de enfermedad en personas mayores, el $92 \%$ de los pacientes priorizan el confort al final de la vida, por lo que debe ser un objetivo relevante de cuidado ${ }^{8}$.

Existe controversia en el ámbito hospitalario o en centros de larga estancia en cuanto al reconocimiento de la demencia en situación de terminalidad o insuficiencia de órgano avanzada, con las dificultades añadidas de comunicación del paciente que obstaculizan la valoración de diferentes síntomas ${ }^{9}$. Una vez identificados los residentes en situación de final de vida o corta expectativa de vida, debe establecerse la proporcionalidad y adecuación de ciertas medidas diagnósticas y terapéuticas, que afectan en gran medida a los cuidados del paciente con demencia avanzada (nutrición enteral en caso de disfagia, utilización de antibióticos ante infecciones de repetición, uso juicioso de traslados hospitalarios, caídas y uso racional de sujeciones físicas y químicas, adecuación farmacológica), y consensuar de forma dinámica junto con el cuidador principal los objetivos asistenciales, centrados en el máximo interés y confort del residente ${ }^{10-13}$.

\section{Objetivos}

El objetivo principal del estudio es describir las variables relacionadas con las necesidades de cuidados al final de la vida de las personas con demencia avanzada en los centros gerontológicos. Los objetivos secundarios de este estudio son:

- Describir las necesidades asistenciales de los residentes con demencia avanzada en situación de final de vida.

- Conocer el grado de confort y control de síntomas.

- Identificar la frecuencia de usuarios que refieren necesidades espirituales.

- Describir la satisfacción de los cuidadores con el proceso de atención recibido.

\section{Pacientes y método}

Estudio descriptivo y prospectivo desde el 1 de julio de 2014 hasta diciembre de 2016, realizado en el centro gerontológico Txara 1 en San Sebastián, que dispone de 120 camas concertadas con la Diputación Foral de Guipúzcoa, gestionado por la Fundación Matia.

El proceso asistencial identificó trimestralmente a aquellos residentes con demencia avanzada en situación de final de vida o de pronóstico de vida limitado, entendido como la presencia de estadio GDS 7 (Global Deterioration Scale) $)^{14}$, junto a la presencia de síndrome declive (deterioro funcional y malnutrición establecida), disfagia con episodios aspirativos, úlceras por presión profundas o múltiples y/o procesos infecciosos de repetición, constituyendo estas características los criterios de inclusión. El criterio de exclusión principal fue la imposibilidad de realizar un seguimiento de los pacientes incluidos, especialmente los ingresos temporales. El periodo de reclutamiento de los residentes ha sido abierto en el tiempo, por lo que el tiempo de seguimiento es variable en función del periodo de inclusión.

Se realizó una entrevista al cuidador principal identificado, informándole del pronóstico y objetivos asistenciales, planificando las posibles decisiones en situación de inestabilidad o descompensación (traslados hospitalarios, nutrición artificial en situación de disfagia grave, adecuación tratamiento farmacológico) y enfatizando la incertidumbre temporal en cuanto a la capacidad de predicción del pronóstico de vida. A las familias se les entregó un folleto informativo, en donde constan los principales problemas que surgen en las personas con demencia avanzada en situación de final de vida ${ }^{15}$.

Para la recogida de variables de estudio se tradujeron las escalas utilizadas en el estudio CASCADE: para el control de sintomas, la escala SM-EOLD (Symptom Management at the End-of-Life in Dementia); para el dolor, dada la dificultad de comunicación presente, se ha utilizado además la escala PAINAD-Sp (Pain Assessment in Advanced Dementia, Spanish version $)^{16-18}$; para el confort en la situación de agonía se utilizó la escala CAD-EOLD (Comfort Assessment in Dying with Dementia), y para medir la satisfacción con el cuidado se utilizó la escala SWC-EOLD (Satisfaction with Care at the End-of-Life in Dementia). Se recogieron las incidencias sanitarias y la utilización de recursos sanitarios que fueron surgiendo en el periodo de estudio.

En caso de aceptar la participación en el estudio, se especificó en la historia clínica electrónica el nivel de intervención adoptado, realizando una monitorización trimestral del control de síntomas (SM-EOLD). En situación de descompensación aguda o de agonía se registró diariamente el grado de confort (CAD-EOLD). Finalmente, tras el fallecimiento, a las 4 semanas se envió una carta de condolencia junto a una encuesta de satisfacción que era remitida con un sobre franqueado (SWC- EOLD).

En situación de agonía, tras un evento gatillo que condiciona el encamamiento del residente o un pronóstico vital de días, se estableció un plan de cuidados específico entre gerocultores y enfermería, incrementando las visitas y priorizando en el plan de cuidados los cuidados de la boca, posturales y de confort. La psicóloga del centro intervino en el cuidado de los aspectos ambientales y de apoyo familiar, informando a todo el equipo interdisciplinar de la situación presente. La trabajadora social identificó situaciones de familias disfuncionales y facilitó a la familia orientación sobre el protocolo a seguir tras el fallecimiento. Desde el punto de vista médico se identificaron y monitorizaron de forma objetiva las necesidades clínicas y el grado de control de las mismas, así como la coordinación de la atención prestada.

De forma periódica se mantuvo contacto con la pastoral diocesana, para que en aquellos residentes católicos se pudiera realizar el sacramento de la unción de enfermos y en situación de agonía, en caso de voluntad de la familia, el sacramento de la extremaunción. Se registraron en la 
historia clínica las necesidades espirituales recogidas en la historia de vida del residente, disponiéndose de un manual de los diferentes credos para abordar globalmente las necesidades integrales del residente ${ }^{19}$. La dimensión espiritual no incluye solo el aspecto religioso, sino todas aquellas creencias, sentimientos que van más allá de lo racional o material, que tienen que ver con componentes que trascienden los fenómenos sensoriales.

El proyecto ha sido avalado por el Comité de Ética Asistencial de la Fundación Matia, con el consentimiento informado de los familiares.

El análisis estadístico se realizó con el programa informático SPSS versión 17.0. La descripción de las variables se hizo mediante porcentajes y frecuencias para variables categóricas. Para variables cuantitativas se informó de la media y desviación típica, del máximo, mínimo y del rango intercuartílico.

\section{Resultados}

En el periodo de estudio se han analizado 74 residentes que cumplen los criterios de inclusión. De los aspectos demográficos de la muestra (Tabla I), destaca lo siguiente: la edad media de los residentes seleccionados es de 87,3 años, siendo mayor en las mujeres; el tiempo de estancia media es de 44 meses desde el inicio de la institucionalización, y la media de medicamentos es de 4,6 fármacos, inferior a la media de los residentes del centro gerontológico, que es de 7,3 fármacos. La etiología principal de la demencia es degenerativa o mixta, seguida de los procesos vasculares.

En cuanto al control de síntomas, se dispone de datos a lo largo de más de 1 año, con una media y desviación típica en cuanto a valoraciones de 3,59 $\pm 2,56$ meses. Es de destacar la importancia de medir el dolor, ya que cuando esto se lleva a cabo se detecta que un $5 \%$ de los pacientes tienen dolor más de una vez a la semana y un $10 \%$ presentan valores superiores a 3 en la escala PAINAD-Sp. Asimismo, los síntomas conductuales y afectivos están presentes en un $12 \%$, como lo muestra la frecuencia de ansiedad o agitación (Tabla II).

A lo largo del tiempo estudiado han fallecido 50 residentes (67 \% de la muestra), con una media y desviación típica de 2,6 \pm 2,69 días de duración (mediana de 3 días), 11 (22\%) de los cuales fallecieron de forma súbita sin permitir registrar el grado de confort (Tabla III). De los resultados de la escala CADEOLD se aprecia la presencia de disconfort en un $40 \%$, dolor en un $20 \%$, y disnea y broncorrea en un $61 \%$, al ser la infección de vías respiratorias bajas la principal causa de fallecimiento. El malestar emocional y la evaluación del bienestar constituyen variables relevantes del confort, con las dificultades de observación y subjetividad inherente dada la frecuencia de delirio hipoactivo presente en los momentos finales de vida. Destaca la existencia de un 7,7 \% de residentes con cierto grado de malestar o sufrimiento en el proceso de final de vida.

En un $66 \%$ de los residentes incluidos se estableció de forma precoz la atención religiosa, en forma de la administración de la unción de enfermos, y en algunos casos se pudo duplicar dicha asistencia en los momentos finales si se dispuso de tiempo para la extremaunción.

Tras el fallecimiento del residente, la encuesta de satisfacción la cumplimentó el cuidador principal en el $30 \%$ de los casos estudiados, observándose un alto grado de satisfacción (Tabla IV).

\section{Discusión y conclusiones}

Este proyecto pionero de atención integral a pacientes con demencia avanzada muestra un adecuado control sintomático en la mayoría de los pacientes en fase de estabilidad, una reducción del número de medicamentos, con un bajo porcentaje de derivaciones y una elevada satisfacción de los familiares.

Existen diferentes instrumentos de cribado que pueden servir para la identificación de pacientes con enfermedad crónica avanzada con un pronóstico de vida limitado, como el instrumento NECPAL (Necesidades Paliativas), ADEPT (Advanced Dementia Prognostic Tool) o el frágil-VIG ${ }^{20-22}$. En todos ellos se tiene en cuenta tanto la gravedad y progresión de la enfermedad, como la utilización de recursos asistenciales. La inclusión de presencia de síndrome de declive y

Tabla I. Características de población estudiada $(n=74)$

Edad (media $\pm \mathrm{DE}): 87,32 \pm 7,62$ años

Hombres: 84,93 (IC95 \% 81,93-87,93)

Mujeres: 87,93 (IC95\% 85,84-90,02)

Sexo: 59 (79,7\%) mujeres, $15(20,3 \%)$ hombres

Estancia (meses $\pm \mathrm{DE}): 44,61 \pm 36,62$

Hombres: 43,47 (IC95 \% 21,28-65,66);

Media recortada $5 \%: 38,36$

Mujeres: 44, 90 (IC95 \% 35,50-54,28);

Media recortada $5 \%: 41,47$

Etiología de la demencia [n (\%)]:

Alzheimer 34 (45,9\%)

Vascular $21(28,4 \%)$

Mixta $8(10,8 \%)$

Cuerpos de Lewy 7 (9,5\%)

Frontotemporal $4(5,4 \%)$

Medicamentos (media $\pm \mathrm{DE}): 4,61 \pm 1,73$

Atención espiritual: 49 (66,2\%)

PAINAD Sp (media \pm DE): 1,34 $\pm 1,60(\leq 3: 90 \% ; 4-6: 8 \%$; $>6: 2 \%)$

Incidencias (media $\pm \mathrm{DE}$ ): 2,86 $\pm 1,24$ (disnea $49 \%$, fiebre $22,4 \%$, dolor $8,2 \%$ )

Utilización de recursos sanitarios en los últimos 6 meses

[n (\%)]:

Urgencias: 6 (12\%)

Ingresos: 13 (26\%)

Un ingreso: 11 (22\%)

Dos ingresos: 2 (4\%)

Causas del fallecimiento $(n=50)$ : IVR $33(66 \%)$, ITU 1

(2\%), sepsis $4(8 \%)$, CV $10(20 \%)$, otros $2(4 \%)$

Localización del fallecimiento: centro gerontológico 47 (94\%), hospital 3 (6\%)

CV: cardiovascular; DE: desviación estándar; ITU: infección del tracto urinario; IVR: infección de las vías respiratorias bajas; PAINAD: Pain Assessment in Advanced Dementia. 
Tabla II. Control de síntomas en residentes con demencia avanzada (SM-EOLD) $(n=74)$

\begin{tabular}{|c|c|c|c|c|c|c|}
\hline & $\begin{array}{c}1 \\
\text { n (\%) }\end{array}$ & $\begin{array}{c}2 \\
\text { n (\%) }\end{array}$ & $\begin{array}{c}3 \\
\text { n (\%) }\end{array}$ & $\begin{array}{c}4 \\
\text { n (\%) }\end{array}$ & $\begin{array}{c}5 \\
\text { n (\%) }\end{array}$ & $\begin{array}{c}6 \\
\text { n (\%) }\end{array}$ \\
\hline Dolor & $54(73)$ & $10(13,5)$ & $6(8,1)$ & $1(1,4)$ & $1(1,4)$ & $2(2,7)$ \\
\hline Respiración acortada & $66(89,2)$ & $6(8,1)$ & $2(2,7)$ & - & - & - \\
\hline Úlceras & $71(95,9)$ & - & - & - & - & $3(4,1)$ \\
\hline Calma & $4(5,4)$ & - & $1(1,4)$ & $4(5,4)$ & $31(41,9)$ & $34(45,9)$ \\
\hline Depresión & $67(90,5)$ & $3(4,1)$ & $1(1,4)$ & $1(1,4)$ & $2(2,7)$ & - \\
\hline Miedo & $66(89,2)$ & $2(2,7)$ & $3(4,1)$ & - & $2(2,7)$ & $1(1,4)$ \\
\hline Ansiedad & $47(63,5)$ & $7(9,5)$ & $11(14,9)$ & $4(5,4)$ & $5(6,8)$ & - \\
\hline Agitación & $39(52,7)$ & $10(13,5)$ & $14(18,9)$ & $8(10,8)$ & $3(4,1)$ & - \\
\hline Resistencia a cuidados & $60(81,1)$ & $4(5,4)$ & $6(8,1)$ & $1(1,4)$ & $2(2,7)$ & $1(1,4)$ \\
\hline
\end{tabular}

1: Nunca. 2: Una vez al mes 3: Dos o tres días al mes. 4: Una vez a la semana. 5: Varios días a la semana. 6: Todos los días. SM-EOLD: Symptom Management at the End-of-Life in Dementia.

Tabla III. Valoración del confort en residentes con demencia al final de la vida (CAD-EOLD) $(n=39)$

\begin{tabular}{|c|c|c|c|}
\hline & $\begin{array}{c}1 \\
\mathrm{n}(\%) \\
\end{array}$ & $\begin{array}{c}2 \\
\mathrm{n}(\%) \\
\end{array}$ & $\begin{array}{c}3 \\
\text { n (\%) } \\
\end{array}$ \\
\hline \multicolumn{4}{|l|}{ Malestar físico } \\
\hline Disconfort & $23(59)$ & $15(38,5)$ & $1(2,6)$ \\
\hline Dolor & $32(82,1)$ & $6(15,4)$ & $1(2,6)$ \\
\hline Inquietud & $21(53,8)$ & $16(4,1)$ & $2(5,1)$ \\
\hline $\begin{array}{l}\text { Respiración } \\
\text { acortada }\end{array}$ & $11(28,2)$ & $23(59)$ & $5(12,8)$ \\
\hline \multicolumn{4}{|c|}{ Síntomas de agonía } \\
\hline Disnea & $15(38,5)$ & $20(51,3)$ & $4(10,3)$ \\
\hline Ahogos & $29(74,4)$ & $9(23,1)$ & $1(2,6)$ \\
\hline Estertores & $21(53,8)$ & $12(30,8)$ & $6(15,4)$ \\
\hline \multicolumn{4}{|c|}{ Malestar emocional } \\
\hline $\begin{array}{l}\text { Dificultad para } \\
\text { tragar }\end{array}$ & $3(7,7)$ & $4(10,3)$ & $32(82,1)$ \\
\hline Miedo & $39(100)$ & $0(0)$ & $0(0)$ \\
\hline Ansiedad & $38(97,4)$ & $1(2,6)$ & $0(0)$ \\
\hline Quejidos & $29(74,4)$ & $7(17,9)$ & $3(7,7)$ \\
\hline Llanto & $36(92,3)$ & $2(5,1)$ & $1(2,6)$ \\
\hline \multicolumn{4}{|l|}{ Bienestar } \\
\hline Serenidad & $3(7,7)$ & $9(23,1)$ & $27(69,2)$ \\
\hline $\mathrm{Paz}$ & $3(7,7)$ & $9(23,1)$ & $27(69,2)$ \\
\hline Calma & $1(2,6)$ & $10(25,6)$ & $28(71,8)$ \\
\hline
\end{tabular}

1: Nunca. 2: A veces. 3: Muchas veces.

CAD-EOLD: Comfort Assessment in Dying with Dementia Scale.

complicaciones asociadas a la inmovilidad identifica, asimismo, a aquellos residentes con insuficiencia orgánica avanzada en la fase final de vida.

Todo ello permite adecuar las terapias farmacológicas al pronóstico (estatinas, antiresortivos óseos, uso racional de inhibidores bomba de protones, hipotensores, antidiabéticos, broncodilatadores, suplementos vitamínicos), lo que constituye un factor relevante de coherencia asistencial, pues provee de ayudas o referencias en la toma de decisiones ${ }^{23,24}$.

Para conseguir la transmisión de dicha información con el cuidador principal debe prepararse adecuadamente la entrevista clínica, y se ha de disponer de tiempo para permitir exponer dudas y establecer una relación horizontal; de esta manera se minimizan los conflictos con las familias al conocer el pronóstico real del residente.

La estructuración de los cuidados, determinando las funciones y responsabilidades de los diferentes miembros del equipo interdisciplinar especialmente al final de la vida, permite cuidar tanto la información como los ambientes, preparando y acompañando a la familia en un momento emocional complejo. La planificación avanzada de cuidados permite adecuar el plan de atención individual a las necesidades integrales del residente, con el apoyo de la familia.

Disponer de escalas de medición orientadas a objetivos prioritarios paliativos, como son el control de síntomas y el grado de confort o bienestar, permite analizar de forma dinámica las necesidades de cada persona, monitorizando la efectividad de los cuidados prestados. Así, la prevalencia de dolor en el estudio CASCADE es del $11 \%$, muy parecida al $10 \%$ de nuestra serie, aunque deben diferenciarse los resultados obtenidos en fase de estabilidad de aquellos que se dan en situación de agonía. De los resultados obtenidos, se identifica que la infección respiratoria aspirativa es la principal causa de fallecimiento y de malestar en los momentos finales de vida, por lo que es imprescindible una adecuada valoración e intervención de la disfagia en los centros gerontológicos, teniendo en cuenta las consideraciones éticas de las diferentes intervenciones ${ }^{25-27}$.

De esta manera se pueden diseñar indicadores de calidad asistencial y herramientas de formación para el personal asistencial, como los disponibles para la atención paliativa en personas con demencia avanzada del programa ACOVE 3 (Assessing Care of Vulnerable Elders) ${ }^{28}$. Asimismo, es importante potenciar, por parte de los cuidadores, unos valores de gran relevancia en el cuidado, como pueden ser la compasión, el respeto, la empatía, el acompañamiento y la disponibilidad. 
Tabla IV. Satisfacción de cuidados en residentes con demencia al final de la vida (SWC-EOLD) $(n=15)$

\begin{tabular}{|c|c|c|c|c|}
\hline Preguntas & 1 & 2 & 3 & 4 \\
\hline 1. Me sentí completamente involucrado en todas las tomas de decisión & - & - & $38 \%$ & $61 \%$ \\
\hline 2. Hubiera tomado posiblemente otra decisión si hubiera tenido más información & $38 \%$ & $46 \%$ & $8 \%$ & $8 \%$ \\
\hline 3. Todas las medidas fueron tomadas para mantener a mi paciente cómodo & - & - & $31 \%$ & $69 \%$ \\
\hline 4. El equipo de salud fue sensible a mis necesidades y sentimientos & - & - & $15 \%$ & $85 \%$ \\
\hline 5. No comprendí la situación de mi receptor de cuidado (paciente) & $30 \%$ & $70 \%$ & - & - \\
\hline 6. Siempre supe lo que el médico o la enfermera hacían para el cuidado del paciente & - & - & $23 \%$ & $77 \%$ \\
\hline 7. Sentí que el receptor de cuidados obtuvo toda la asistencia de enfermería que precisó & - & - & $31 \%$ & $69 \%$ \\
\hline 8. Sentí que se explicaron con claridad todos los aspectos médicos & - & - & $15 \%$ & $85 \%$ \\
\hline $\begin{array}{l}\text { 9. El receptor de cuidados recibió todos los tratamientos e intervenciones de las que } \\
\text { se podría haber beneficiado }\end{array}$ & - & - & $38 \%$ & $61 \%$ \\
\hline 10. Siento que necesitó mejor atención médica al final de su vida & $54 \%$ & $46 \%$ & - & - \\
\hline
\end{tabular}

1: Muy en desacuerdo. 2: En desacuerdo. 3: De acuerdo. 4: Muy de acuerdo.

SWC-EOLD: Satisfaction with Care at the End-of-Life in Dementia.

La identificación de residentes con demencia avanzada en situación de final de vida tiene una gran importancia tanto a nivel comunitario como residencial, debiendo quedar constancia de ello en la historia clínica. Esto permite que, en caso de acudir a un recurso externo al centro ante una descompensación aguda, estén especificados claramente los objetivos asistenciales. De esta manera se consigue una prevención de traslados innecesarios, falleciendo un $94 \%$ de los residentes incluidos en el mismo centro. Aunque lo deseable sea que el paciente en situación de últimos días fallezca en el centro gerontológico, esto no siempre es posible tanto por condicionantes del propio paciente o familia, como inherentes a la tipología de los centros y a su dotación de profesionales, y este hecho no ha de ser vivido como un fracaso por el propio equipo.

Los centros gerontológicos no suelen entrar en los planes de salud de las diferentes comunidades, que se centran más en la atención y coordinación comunitaria y especializada, siendo recursos sociales con una alta carga sanitaria en donde la atención paliativa tiene un importante papel ${ }^{29}$.

Un reto añadido es la verificación del familiar identificado como interlocutor con el centro, si realmente es garante de los intereses y valores del residente incompetente, ya que en pocas ocasiones se dispone de un tutor legal, por lo que, tras el diagnóstico de un deterioro cognitivo o demencia instaurada, debería establecerse un interlocutor válido que responda adecuadamente en la protección de la persona.

Las encuestas de satisfacción disponibles son positivas, aunque existe un sesgo de selección claro al contestar posiblemente los que más agradecidos o satisfechos están con los cuidados. También es posible que la carta que se envía con sello franqueado para su contestación no sea el mejor método, por lo que se están desarrollando otros como la llamada telefónica.

Una limitación del estudio es el tamaño muestral o la ausencia de grupo control, así como los diferentes tiempos de seguimiento; sin embargo, la metodología utilizada permite conocer y monitorizar de forma dinámica las trayectorias de final de vida.
Como se puede ver, lo importante en los momentos finales de la vida no es tanto el "cuándo" o el "donde", sino más bien el "cómo", buscando el máximo confort y bienestar de la persona.

\section{Financiación y conflicto de intereses}

No existe ninguna fuente de financiación para el proyecto, ni conflictos de interés.

\section{Agradecimientos}

Agradecemos a las familias y a la pastoral su implicación en el abordaje integral de las personas al final de su vida, y a todo el personal cuidador del centro por su encomiable labor.

\section{Bibliografía}

1. Estadísticas sobre residencias: distribución de centros y plazas residenciales por provincia. Datos de diciembre de 2013. Madrid, Informes en Red, $n^{\circ}$ 7. [Fecha de publicación: 0/02/2014]. Disponible en: http://envejecimiento.csic.es/documentos/ documentos/enred-estadisticasresidencias2013.pdf

2. Martínez-Lage P, Martín-Carrasco M, Arrieta E, rodrigo J, Formiga F. Mapa de la enfermedad de Alzheimer y otras demencias en España. Proyecto MapEA. Rev Esp Geriatr Gerontol. 2018;53:26-37.

3. Mitchell S, Kiely D, Hamel MB. Dying with advanced dementia in the nursing home. Arch Intern Med. 2004;164:321.

4. Mitchell S, Kiely D, Jones RN, Prigerson H, Volicer L, Teno JM. Advanced dementia research in the nursing home: The CASCADE Study. Alzheimer Dis Assoc Disord. 2006;20:166-75.

5. Engel S, Kiely D, Mitchell S. Satisfaction with end-of-life care for nursing home residents with advanced dementia. J Am Geriatr Soc. 2006;54:1567-72.

6. Mitchell S, Teno J, Kiely D, Shaffer ML, Jones RN, Prigerson HG, et al. The clinical course of advanced dementia. N Engl J Med. 2009;361:1529-38. 
7. Mitchell S, Black B, Ersek M, Hanson LC, Miller SC, Sachs GA, et al. Advanced dementia: State of the art and priorities for the next decade. Ann Intern Med. 2012;156:45-51.

8. Bischoff KE, Sudore R, Miao Y, Boscardin WJ, Smith AK. Advance care planning and the quality of end-of-life care in older adults. J Am Geriatr Soc. 2013;61:209-14.

9. Hendriks SA, Smalbrugge M, Hertogh CM, Van Der Steen JT. Dying with dementia: symptoms, treatment, and quality of life in the last week of life. J Pain Symptom Manag. 2014;47:710-20.

10. American Geriatrics Society. 2013. Choosing wisely. Ten things clinicians and patients should question. Disponible en: http:// www.choosingwisely.org/societies/american-geriatrics-society/

11. Alagiakrishnan K, Bhanji R, Kurian M. Evaluation and management of oropharyngeal dysphagia in different types of dementia: A systematic review. Arch Gerontol Geriatr. 2013;56:1-9.

12. American Geriatrics Society Ethics Committee and Clinical Practice and Models of Care Committee. American Geriatrics Society Feeding tubes in advanced dementia position statement. J Am Geriatr Soc. 2014;62:1590-3.

13. Rexach L. Palliative care in dementia. Eur Geriatr Med. 2012;3:131-40.

14. Reisberg B, Ferris SH, de León MJ, Crook T. The global deterioration scale for assessment of primary degenerative dementia. Am J Psychiatry. 1982;139:1136-9.

15. Arcand M, Caron Ch. Comfort care at the end of life for persons with Alzheimer's Disease or other degenerative diseases of the brain. A Guide for Caregivers. Institute Universitarie de Gériatrie de Sherbrooke; 2005.

16. Warden V, Hurley AC, Volicer L. Development and Psychometric Evaluation of the Pain Assessment in Advanced Dementia (PAINAD) Scale. J Am Med Dir Assoc. 2003;4:9-15.

17. García-Soler A, Sánchez-Iglesias I, Buiza C, Alaba J, Navarro $A B$, Arriola $E$, et al. Adaptación y validación de la versión española de la escala de evaluación de dolor en personas con demencia avanzada: PAINAD-Sp. Rev Esp Geriatr Gerontol. 2014;49:1-46.

18. Alaba J, Arriola E, Navarro A, González MF, Buiza C, Hernández C, et al. Demencia y dolor. Rev Soc Esp Dolor. 2011;3:176-86.

19. Sobrevia X, Torradeflot F. Atención religiosa al final de la vida. Conocimientos útiles sobre creencias y convicciones. Obra social "La Caixa"; 2013.

20. Gómez-Batiste X, Martínez-Muñoz M, Blay C, Amblàs J, Vila L, Costa X, et al. Proyecto NECPAL CCOMS-ICO ${ }^{\circ}$ : Identificación y
Atención Integral-Integrada de Personas con Enfermedades Crónicas Avanzadas en Servicios de Salud y Sociales. Centro Colaborador de la OMS para Programas Públicos de Cuidados Paliativos. Institut Català d'Oncologia. Disponible en: http:// ico.gencat.cat/web/.content/minisite/ico/professionals/documents/qualy/arxius/doc_necpal_ccoms-ico_instrumento_esp_ vweb_vff.pdf

21. Mitchell S, Millar S, Teno J, Kiely DK, Davis RB, Shaffer ML. Prediction of 6-month survival of nursinghome residents with advanced dementia using ADEPT vs Hospice Eligibility Guidelines. JAMA. 2010;304:1929-35.

22. Amblás-Novellas J, Martori JC, Molist Brunet N, Oller R, GómezBatiste X, Espaulella Panicot J. Índice frágil-VIG: diseño y evaluación de un índice de fragilidad basado en la Valoración Integral Geriátrica. Rev Esp Geriatr Gerontol. 2017;52:119-27.

23. Holmes H, Sachs G, Shega J, Hougham GW, Cox Hayley D, Dale W. Integrating palliative medicine into the care of persons with advanced dementia: identifying appropriate medication use. J Am Geriatr Soc. 2008;56:1306-11.

24. Tjia J, Rothman M, Kiely D, Shaffer ML, Holmes HM, Sachs GA, et al. Daily medication use in nursing home residents with advanced dementia. J Am Geriatr Soc. 2010;58:880-8.

25. American Geriatrics Society Ethics Committee and Clinical Practice and Models of Care Committee. American Geriatrics Society Feeding Tubes in Advanced Dementia Position Statement. J Am Geriatr Soc. 2014;62:1590-3.

26. Alagiakrishnan K, Bhanji RA, Kurian M. Evaluation and management of oropharyngeal dysphagia in different types of dementia: A systematic review. Arch Gerontol Geriatr. 2013;56:1-9.

27. Volkert D, Chourdakis M, Faxen-Irving G, Frühwald T, Landi F, Suominen $\mathrm{MH}$, et al. ESPEN guidelines on nutrition in dementia, Clinical Nutrition (2015). Disponible en: http://dx.doi. org/10.1016/j.clnu.2015.09.004.

28. Wenger N, Solomon D. Application of assessing care of vulnerable elders-3 quality indicators to patients with advanced dementia and poor prognosis. J Am Geriatr Soc. 2007;55:5457S463.

29. Van der Steen JT, Radbruch L, Hertogh C, de Boer ME, Hughes JC, Larkin P, et al., on behalf of the European Association for Palliative Care (EAPC). White paper defining optimal palliative care in older people with dementia: A Delphi study and recommendations from the European Association for Palliative Care. Palliat Med. 2014;28:197-209. 